\title{
The Pseudo-Observation Analysis of Time-To-Event Data. Example from the Danish Diet, Cancer and Health Cohort Illustrating Assumptions, Model Validation and Interpretation of Results
}

\author{
${ }^{1}$ Aalborg University Hospital, Department of Cardiology Aalborg AF Study Group, Aalborg, Denmark, E-mail: Imm@ph.au.dk \\ ${ }^{2}$ Aarhus University, Department of Public Health, Section for Epidemiology, Aarhus, Denmark, E-mail: Imm@ph.au.dk \\ ${ }^{3}$ Aarhus University, Department of Public Health, Section for Biostatistics, Aarhus, Denmark
}

\begin{abstract}
:
Regression analyses for time-to-event data are commonly performed by Cox regression. Recently, an alternative method, the pseudo-observation method, has been introduced. This method offers new possibilities of analyzing data exploring cumulative risks on both a multiplicative and an additive risk scale, in contrast to the multiplicative Cox regression model for hazard rates. Hence, the pseudo-observation method enables assessment of interaction on an additive scale. However, the pseudo-observation method implies more strict model assumptions regarding entry and censoring but avoids the assumption of proportional hazards (except from combined analyses of several time intervals where assumptions of constant hazard ratios, risk differences and relative risks may be imposed). Only few descriptions of the use of the method are accessible for epidemiologists. In this paper, we present the pseudo-observation method from a user-oriented point of view aiming at facilitating the use of this relatively new analytical tool. Using data from the Diet, Cancer and Health Cohort we give a detailed example of the application of the pseudo-observation method on time-to-event data with delayed entry and right censoring. We discuss model control and suggest analytic strategies when assumptions are not met. The introductory model control in the data example showed that data did not fulfill the assumptions of the pseudo-observation method. This was caused by selection of healthier participants at older baseline ages and a change in the distribution of study participants according to outcome risk during the inclusion period. Both selection effects need to be addressed in any time-to-event analysis and we show how these effects are accounted for in the pseudo-observation analysis.

The pseudo-observation method provides us with a statistical tool which makes it possible to analyse cohort data on both multiplicative and additive risk scales including assessment of biological interaction on the risk difference scale. Thus, it might be a relevant choice of method - especially if the focus is to investigate interaction from a public health point of view.
\end{abstract}

Keywords: time-to-event analysis, pseudo-observation method, model control, risk model, risk difference additivity, interaction

DOI: 10.1515/em-2017-0015

Received: September 22, 2017; Revised: April 19, 2018; Accepted: May 3, 2018

\section{Introduction}

Time-to-event analyses are often used in epidemiological research. Primarily two main analytical methods have been used: this is the Kaplan-Meyer estimator to obtain unadjusted estimates of cumulative risks - or the AalenJohansen estimator in studies with competing risks - and Cox proportional hazards regression (Cox 1972) that operates on a multiplicative scale in a rate model to estimate unadjusted and adjusted hazard ratios (HR). Delayed entry and censoring are easily handled in both the Kaplan-Meyer estimator, the Aalen-Johansen estimator, and in the Cox regression. Alternative methods such as additive hazard models, the Fine and Grey 
regression model and parametric time-to-event models exist (Fine and Gray 1999; Martinussen and Scheike 2006; Cleves et al. 2010; Rod et al. 2012) but are rarely used in practice. A relatively new method, the pseudoobservation method (Andersen and Perme 2010; Parner and Andersen 2010; Overgaard, Andersen, and Parner 2015), is an alternative approach which - like the Cox regression - makes it possible to perform multivariate analyses of right censored data with delayed entry, but - in contrast to Cox regression - allows calculation of both relative and absolute measures of association. With the introduction of the pseudo-observation method, a gap was filled in the analysis opportunities of time-to-event studies enabling multivariate regression analysis in an absolute risk model (Table 1). We acknowledge that the Fine and Gray model, which is also based on a risk model, has been around for a while, however the interpretation of the parameters is not straight forward (Andersen and Keiding 2012).

Table 1: Analyses tools for time-to-event data after the introduction of the pseudo-observation method.

\begin{tabular}{lll}
\hline & Risk model & Rate model \\
\hline One possible event & $\begin{array}{l}\text { Kaplan-Meyer } \\
\text { (univariate analysis) } \\
\begin{array}{l}\text { Pseudo-observation method } \\
\text { (multivariate analysis) }\end{array}\end{array}$ & $\begin{array}{l}\text { Cox regression } \\
\text { (multivariate analysis) }\end{array}$ \\
\hline Several possible events ${ }^{\mathrm{a}}$ & $\begin{array}{l}\text { Aalen-Johansen } \\
\text { (univariate analysis) } \\
\begin{array}{l}\text { Pseudo-observation method } \\
\text { (multivariate analysis) }\end{array}\end{array}$ & $\begin{array}{l}\text { Cox regression } \\
\text { (multivariate analysis) }\end{array}$ \\
\hline
\end{tabular}

a Assuming the occurrence of other events prevents the occurrence of the event of interest, i. e. competing risks.

\subsection{Aim and demonstration study}

In this expository paper, we present the pseudo-observation method in order to facilitate the use of it and to provide a description of the assumptions, model validation and interpretation of results. This is done using data from the Danish Diet, Cancer and Health Cohort (Tjonneland et al. 2007), where we applied the pseudoobservation method in a study of dietary intake of long-chain polyunsaturated fatty acids (LC-PUFAs) and risk of atrial fibrillation (AF). The LC-PUFAs consist of two main groups, the n-3 and n-6. Both groups are involved in the regulation of systemic inflammation which has been shown to be associated with AF (Wu et al. 2013), but the inflammatory effects from n-6 LC-PUFAs are stronger than the effects from n-3, thus n-3 are said to be relatively anti-inflammatory and n-6 pro-inflammatory. Further, upon digestion, the two PUFA groups are metabolized in the same biochemical pathway, where they share enzymes in a competitive way. Therefore, the intake level of n-3 might influence the effect of n-6 on risk of AF and vice versa. Due to this expected interplay, the key aim of this study was to explore interaction between intake of n-3 and n-6 LC-PUFA and risk of AF. The analysis presented here is based on a simplified model which serves as an illustrative example to demonstrate the application of the method. The full analysis of data has been published elsewhere (Mortensen et al. 2017).

Assessment of interaction: There is an ongoing discussion about whether the underlying statistical model should be additive or if relative measures from a multiplicative model is useful for evaluation of biological interaction (Rothman, Greenland, and Walker 1980; Ahlbom and Alfredsson 2005; VanderWeele 2009; Lawlor 2011; Andersen and Skrondal 2015). This discussion is beyond the scope of the present paper. Based on Rothmans "sufficient cause diagrams" (Greenland, Lash, and Rothman 2008), we decided to analyse the biologically substantiated potential interaction as deviation from additivity of absolute measures of association and thus the HR, as a relative measure of association, provided in the Cox model, would be an obvious limitation.

\section{The pseudo-observation method}

The pseudo-observation method includes two consecutive analytical parts: The generation of the pseudoobservations and the regression analysis. The first step, the generation of the pseudo-observations, relies on a transformation of data based on the Kaplan-Meier/Aalen-Johansen estimator for a predefined time interval (a start and an end time point) resulting in one set of pseudo-observations. In case of one time interval, 
one pseudo-observation is generated for each participant in the data set specific for that time interval (this is what has been done in the present study). If more than one time interval is analyzed there will be a set of pseudo-observations for each time interval (Parner and Andersen 2010). The transformation of the data into pseudo-observations enables the data set to be analyzed using a generalized linear model in spite of the presence of censoring. The pseudo-observations estimate the individual event status at a specified time point had there not been censoring in the cohort. In the unlikely situation of a time-to-event data set without censoring, the pseudo-observation would be the indicator of an event at the specific time point and this could be analyzed in a simple binomial risk model. Due to the inevitable censoring, the transformation step is needed.

The second step is the generalized linear modeling of the pseudo-observations. The regression included in the generalized linear modeling is flexible regarding choice of scale, and the method allows us to use both relative and absolute measures of association as well as restricted mean and the life-time lost due to a specific cause of death.

The underlying mathematics in the transformation of data and in the generalized linear modeling are described elsewhere (Parner and Andersen 2010).

\subsection{Elements of the model validation of the pseudo-observation method}

The pseudo-observation method includes two steps. This also applies to the model control: The validation of (I) the generation of the pseudo-observations, and (II) the generalized linear model including the correct modeling of the covariates. The principles behind the model assumptions and how violations can be tackled are described below.

\section{Validation of the pseudo-observations}

Assumptions of independency. In time-to-event analyses, it is essential that the cohort is representative of a target population - not only at the time of enrolment - but also throughout the follow-up period. We use the term continuous representativeness to describe that the cohort - in spite of changes over time - still represents the target population. It should be noted that we here define target population conservatively as the population represented by the persons who agreed to participate in the cohort.

To ensure the continuous representativeness of cohorts, two points in time are particularly important: 1) at baseline when the follow-up is initiated; and 2) when the follow-up is ended for other reasons than an event (e. g. censoring caused by loss to follow-up or administrative end of follow-up). Regarding item 1, the critical variables are baseline age and baseline calendar time in cohort designs where these vary. Even though random recruitment during the inclusion period (baseline calendar time) and across different baseline ages is intended when establishing a cohort, it must be checked if the sampling is in fact random. For example in a time-to-event study where current age is used as the time scale in the analysis (as in the present example), the subjects in the cohort must have similar event risks (hazards) at a given age in the analysis regardless of their age at entry in the study (baseline age). This principle is illustrated in Figure 1A and is referred to as the independent entry assumption (termed assumption 1 in this paper). Similarly, and this relates to item 2, those censored from the cohort at a given age should have the same event risk as those still at risk at that age. This principle is illustrated in Figure 1B and is referred to as the independent censoring assumption (Greenland, Lash, and Rothman 2008; Andersen and Keiding 2012) (termed assumption 2 in this paper).
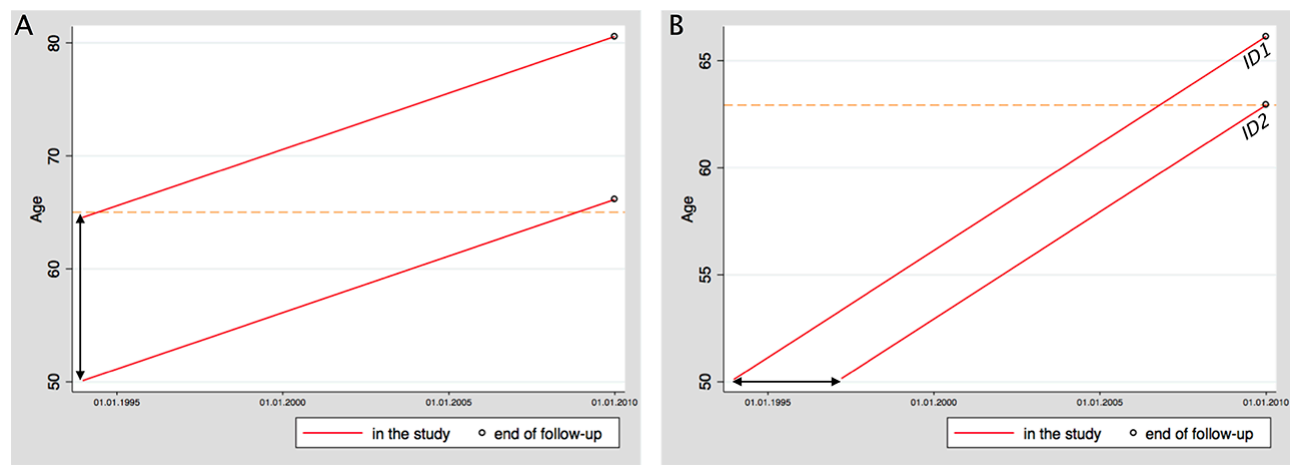
Figure 1: Lexis diagrams each illustrating two participants in the Diet, Cancer and Health cohort (Denmark, 1993-1997). In $\mathrm{A}$, the participation of two subjects with low and high baseline age are outlined (red lines). The vertical double-headed arrow shows the baseline age span in the cohort. The yellow, dotted line illustrates an age, where the two persons are compared in an analysis of the full cohort with current age used as underlying time axis. In order to meet assumption 1 , the two participants must have the same AF risk in spite of the difference in baseline age. This was not the case, and therefore the dataset had to be stratified. In B, two subjects with low and high baseline calendar time are outlined (red lines). The span in baseline calendar time in the cohort is illustrated by a double-headed arrow. According to assumption 2 , the different baseline calendar time must be independent of AF risk during follow-up. Thus, the person (ID2) censored administratively at the age of 63 years must have the same AF risk as the person (ID1), who stays in the study at age 63 due to an earlier baseline calendar time.

The validity of all time-to-event analyses with delayed entry and censoring hinges on the two assumptions regarding independence. In Cox regression, the two general assumptions concerning independency are conditioned on the covariates in the model allowing adjustment for these variables before testing for potential violations in the model control phase. In contrast, the independency assumptions for the pseudo-observation method are more restrictive because the generation of the pseudo-observations relies on the Kaplan-Meierestimator/Aalen-Johansen-estimator to be valid, and neither of these estimators takes covariates into account. Thus, the independency assumptions have to be met without conditioning on covariates, meaning that these assumptions are stricter than in the adjusted Cox models. Due to this narrowing of the basic assumptions, the independent censoring assumption has been termed completely independent censoring assumption when applied to the pseudo-observation method (Overgaard, Andersen, and Parner 2015). Likewise, this terminology applies to the completely independent entry assumption (unconditional independence between entry and event risk). Furthermore, in the pseudo-observation method, the entry distribution (baseline age and baseline calendar time) and censoring should be independent of the covariates in the generalized linear model (Overgaard, Parner, and Pedersen 2017)(referred to as assumption 3 and 4 in this paper) Although there seems to be redundancy between the assumptions of complete independency between entry/censoring and event risk (assumption 1 and 2 ) and assumptions concerning independency between entry/censoring and covariates (assumption 3 and 4), it is not possible to deduce one assumption from the other. Therefore, it is necessary in the pseudo-observation method to check all four assumptions regarding independency.

\section{Validation of the generalized linear model}

The generalized linear model covers a large number of regression types, which are specified by the distribution of the outcome and a link function connecting the mean outcome with the linear combination of the explanatory variables. The most common regression analyses are multiple linear regression, logistic regression, other binary regression (to estimate risk difference or risk ratio) and Poisson regression. In any case, this part of the model control must be performed based on the same principles as the validation of a binary regression model. Thus, the relevant assumptions are: Linearity of continuous covariates and no interaction between covariates. Further, in a combined analysis of multiple time intervals, an additional assumption of constant hazard ratio (or relative risk or risk difference depending on the measure of association) may be imposed. This situation will not be addressed further in this paper.

2.

An outline of the assumptions behind the pseudo-observation method and Cox regression is shown in Table

Table 2: Assumptions in Cox regression and the pseudo-observation method.

\begin{tabular}{|c|c|c|}
\hline Assumptions & Cox regression & $\begin{array}{l}\text { Pseudo-observation } \\
\text { method }\end{array}$ \\
\hline \multicolumn{3}{|l|}{ Assumptions related to independency } \\
\hline $\begin{array}{l}\text { Assumption } 1 \text { (The independent entry assumption): } \\
\text { Entry is independent of event risk }\end{array}$ & $\begin{array}{l}\text { Yes } \\
\text { (conditioned on covariates) }\end{array}$ & $\begin{array}{l}\text { Yes } \\
\text { (un-conditioned) }\end{array}$ \\
\hline $\begin{array}{l}\text { Assumption } 2 \text { (The independent censoring assumption): } \\
\text { Censoring is independent of event risk }\end{array}$ & $\begin{array}{l}\text { Yes } \\
\text { (conditioned on covariates) }\end{array}$ & $\begin{array}{l}\text { Yes } \\
\text { (un-conditioned) }\end{array}$ \\
\hline Assumption 3: Entry time is independent of covariates & No & Yes \\
\hline Assumption 4: Censoring is independent of covariates & No & Yes \\
\hline
\end{tabular}

Assumptions related to the regression

Linearity of continuous covariates

Yes

Yes 
Yes Yes

\subsection{How to handle violations of the independency assumptions}

If the time-to-event data fail to meet the assumptions, the solution presented here is to split the data into strata according to levels of the problematic variable (e. g. baseline age) (Andersen and Perme 2010; Hansen, Andersen, and Parner 2014; Andersen, Syriopoulou, and Parner 2017). If, for example, assumption 1 is violated in terms of an association between baseline age and the event in question, the data set must be stratified according to baseline age (e. g. tertiles, quartiles or quintiles depending on the actual data set, as discussed below), and it has to be assessed in each stratum if the association has diminished to an acceptable level. If so, the further model control is carried out within each strata and so is the generation of pseudo-observations, provided that they fulfill all assumptions.

This part of the model control is an iterative, stepwise process resulting in a final set of strata which all meet the assumptions. In the process, the statistical analyses and tests are used to identify general patterns and trends in associations and this interpretation is not strictly based on statistical significance. The assessments, compromises and decisions along the various steps of the model control depend on the data set in question, and during this process, it is vital that the refinement of the model does not involve testing and quantifying the association between the primary exposure and the outcome. Further, according to the principles presented by Harrell (2001), we also argue that exploring associations between covariates and the outcome is not part of the model refinement. Hence, the strata structure based on the assumptions has to be pre-specified and not driven by the outcome distribution, but rather a reasonable stratification into tertiles, quartiles or quintiles or other predefined strata structures depending of cohort size and the specified research question. Data driven adjustments during the iterative process may be necessary, but large changes must be accounted for. As a general rule, the larger strata, the better precision of the estimates. This calls for as few strata as possible. Further requirements and considerations regarding stratification are discussed in Section 2.4.

\subsection{Strata definitions}

When generated, the strata specific pseudo-observations can either be combined to obtain an analysis of the entire data set, or regression analyses can be conducted for each stratum. In order to combine strata, the time frames for the cumulative risks (i. e. the start- and end times, e. g. age 50 and 65 years), which are encoded in the same step as the pseudo-observations, must be identical. If the strata do not have the same start- and end time points, the risk periods estimated in the pseudo-observations are not comparable and therefore it will not make sense to combine them.

We suggest two terms to cover different strata definitions in the pseudo-observation method. As is normal practice in epidemiology, we suggest the term 'stratum' to denote the strata in which the data set is permanently divided throughout the analyses resulting in separate measures of association for each stratum. In contrast, we suggest 'pseudo-observation stratum' to cover the intermediate sub-strata which are combined before the regression analyses are conducted. An indispensable prerequisite to pseudo-observation strata (in order to be collapsible) is that they are encoded with identical time intervals for cumulative risk, and this criterion distinguishes "strata" from "pseudo-observation strata".

\subsection{Sufficient coverage of the analysis period within each stratum}

In addition to the assumptions described, there also have to be enough participants at risk at appearance of each case when calculating cumulative risk using Kaplan-Meier/Aalen-Johansen. To prevent that imprecise calculated risks affect the total cumulative risk estimate resulting in wide confidence intervals, it must be checked how well the selected periods (age, calendar time, time-on-study) are covered in each pseudo-observation stratum (or in the full data set in case of no stratification). This can be assessed simply by counting the number 'at risk' at appearance of the first and the last event in each stratum since the amount of data would be most sparse here. No objective rules specify the number at risk needed at appearance of each case, but KaplanMeyer/Aalen-Johansen plots with confidence intervals make it possible to visualize the necessary number at risk. Although it is related, it is not directly a result of the number of participants in the cohort, but more of the temporal distribution of cases and persons at risk. However, the larger strata the more likely it is to have enough persons at risk at appearance of each case. Thus, this requirement also calls for a limitation of the number of pseudo-observation strata. 
Apart from the requirement for temporal distribution of cases, several simulation studies have shown that the pseudo-observation method has very good small-sample qualities (10 events per variable are needed in regression analysis for risk difference estimation and 15 events per variable are needed for relative risks) (Hansen et al. 2014).

\section{Application of the pseudo-observation method on the demonstration study}

The demonstration study: Intake of n-3 and n-6 LC-PUFAs and risk of atrial fibrillation: analysis of potential interaction in an additive risk model

\subsection{Study population, exposure and outcome}

The Danish Diet, Cancer and Health cohort contains data from 57,053 men and women (Tjonneland et al. 2007). Participants aged 50 to 65 years were enrolled from December 1993 to May 1997. Thus, both time-related baseline variables varied. Due to non-random sampling during the inclusion period, baseline calendar time was associated with covariates. This was also the case for baseline age which was furthermore associated with risk of AF. The observation period was administratively ended on December 30, 2009. After exclusion of participants with a history of AF, atrial flutter (AFL), cancer, myocardial infarction (MI), or heart failure (HF) at baseline or missing covariates, the final study population totaled 54,737 participants. The median follow-up time was 13.5 years. The primary exposures, intake of n-3 and n-6 LC-PUFA, were estimated based on food frequency questionnaires. The outcome, denoted AF throughout this article, was incident AF and/or AFL during follow-up without preceding MI or HF. Diagnoses were extracted from the Danish National Patient Registry by cross-linking civil registration numbers.

\subsection{Statistical analysis}

Data were analyzed using the pseudo-observation method. In order to estimate the cumulative risks in age intervals (opposed to calendar time intervals or time-on-study), age was chosen as the underlying time scale; accordingly data were analyzed with delayed entry and time zero was the age at birth. We denote the age scale in the time-to-event analysis for current age in order to distinguish it from baseline age. The decisions regarding time scale also applied to the Cox regressions in the model control. People were treated as 'at-risk' until emigration, change of personal identification number, MI, HF, death, AF-diagnose or administrative end of follow-up. A diagnose of AF (as defined above) was the outcome and death, MI and HF during follow-up were treated as competing risks. In order to assess interaction as departure from additivity of the measures of association, the primary exposures were modeled categorically, thus, the intake of n-3 and n-6 LC-PUFA were expressed in tertiles resulting in 9 exposure groups in the interaction analysis. The lowest tertile group according to both exposures was used as reference. A simplified model was used adjusting for baseline information on hypertension (as an example of a categorical confounder) and BMI (as an example of a continuous confounder). Results were expressed as risk differences.

The statistical model explored the association between n-3 and n-6 and risk of AF adjusted for hypertension and BMI estimated as risk differences in 15-year periods of cumulative risk: Between the age of 50-65 years, 55-70 years and 60-75 years in three separate strata defined by baseline age tertiles given no AF by the start time. The rationale for the stratification is given in 3.4

$$
\begin{aligned}
& \text { Model }_{\text {stratum 1 }}: \operatorname{Pr}(\text { AF at } 65 \mathrm{y} \mid \text { no AF by } 50 \text { y }) \sim n-3 * n-6+\text { hypertension }+\mathrm{BMI}_{\text {spline }} \\
& \text { Model }_{\text {stratum 2 }}: \operatorname{Pr}(\text { AF at } 70 \mathrm{y} \mid \text { no AF by } 55 \mathrm{y}) \sim \mathrm{n}-3 * \mathrm{n}-6+\text { hypertension }+\mathrm{BMI}_{\text {spline }} \\
& \text { Model }_{\text {stratum 2 }}: \operatorname{Pr}(\mathrm{AF} \text { at } 75 \mathrm{y} \mid \text { no AF by } 60 \mathrm{y}) \sim \mathrm{n}-3 * \mathrm{n}-6+\text { hypertension }+\mathrm{BMI}_{\text {spline }} \\
& \text { * denotes an interaction between the variables. }
\end{aligned}
$$

Data were analyzed using Stata Statistical Software (Stata 13) (StataCorp 2013). 


\subsection{Test of the assumptions}

The assumptions are presented separately below. For each assumption, a general definition is followed by a specific description of the analysis carried out in the actual data example. This part of the text is set with monospace font. We have consistently modelled baseline age and recruitment date categorically (as tertiles). This choice seemed reasonable as this would be in line with a possible stratification (in case of violations of the assumptions), but these variables can also be modelled differently (e.g. as quintiles or continuous). The important thing is that overall patterns and trends in the associations are revealed. Please be aware that current age in the time-to-event analysis and baseline age are not the same as this is important for the interpretation of the assumptions.

\section{Assumption 1}

'The independent entry assumption' implies that the event risk must be independent of entry which is determined by choice of time scale in the actual study.

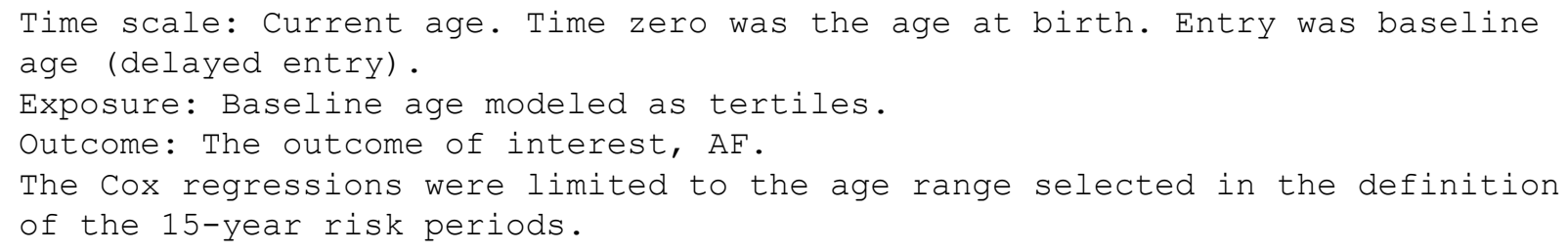

\section{Assumption 2}

'The independent censoring assumption' implies that the event risk must be independent of the time of censoring. In analyses with age as the time scale, the relevant time unit in assumption 2 is age at censoring. The types of censoring and whether these are testable or not, differ by various study designs. The independence of censoring due to loss to follow-up (e. g. emigration) cannot be tested using solely the information available in the time-to-event data whereas some types of administrative censoring can. In designs with administrative censoring at a fixed date (as in the present example), age at this censoring date can be tested by taking into account that age at the administrative censoring $\left(\mathrm{age}_{\mathrm{admin}}\right)$ is a function of the date at administrative censoring $\left(\right.$ date $\left._{\text {admin }}\right)$, and baseline age $\left(\right.$ age $\left._{\text {baseline }}\right)$ and date at baseline $\left(\right.$ date $\left._{\text {baseline }}\right)$ :

$$
\text { age }_{\text {admin }}=\text { age }_{\text {baseline }}+\left(\text { date }_{\text {admin }}-\text { date }_{\text {baseline }}\right)
$$

Evaluation of the testable part of assumption 2 involves the three terms on the right side of the equation. When all terms are independent of AF risk, so is the age at administrative censoring.



It is important to put emphasis on this link between the assumptions as the information from the test of assumption 1 is a prerequisite for completing the test of assumption 2, according to eq. (1).

\section{Assumption 3}

'The distribution of entry time is independent of covariates' 


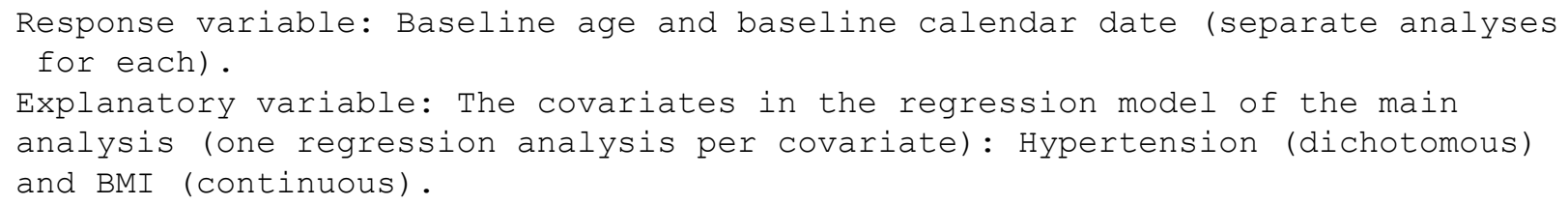

\section{Assumption 4}

'The distribution of time of censoring is independent of covariates'

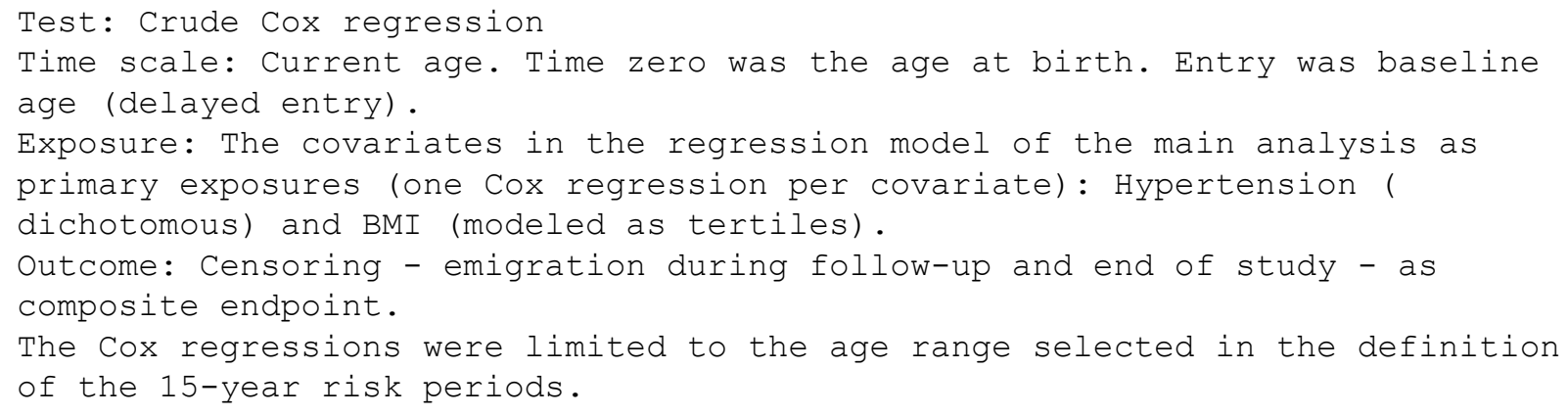

Where assumptions 1 and 2 were focused on entry and censoring in relation to event risk, assumptions 3 and 4 were dedicated to entry and censoring in relation to covariates. Covariates are, per definition, risk factors for the event. Therefore, given independency between entry and censoring and risk of event (addressed in assumptions 1 and 2), we can also expect independency between entry/censoring and covariates. In this way, assumptions 3 and 4 are related to assumptions 1 and 2. If 1 and 2 are fulfilled, the covariates should be independent of both entry and censoring because of the association between the covariates and event risk. However, in circumstances where time trends in distribution of covariates counterbalance each other in their associations with event risk, these trends will not be captured in the assessments of the completely independency assumptions. Thus, all four assumptions are required.

\section{The generalized linear model}

Departures from linearity can be explored for each covariate by testing if the coefficients of cubic spline function deviate significantly from 0 (Wald test). Likewise, adding a product term to the model can test the interaction between covariates (Wald test). However, in the present example, a test of linearity of covariates was unnecessary because the only continuous variable, BMI, was modeled as a spline as suggested by Andersen and Perme (2010). For simplicity of the model, we did not test for interaction between hypertension and BMI.

\subsection{The stepwise model validation process based on the independency assumptions}

The risk of AF during follow-up had to be independent of baseline age and baseline calendar time, according to assumption 1 and 2 . The span in these variables and the follow-up period is illustrated in Figure 1 (the doubleheaded arrows).

The strata structure: The starting point was that baseline age was associated with the development of AF, meaning that assumption 1 was violated (as illustrated in Figure 1A).

The first step in handling this was to stratify the study population into baseline age tertiles, resulting in strata 1,2, and 3. An overview of the three strata is presented in Figure 2. 


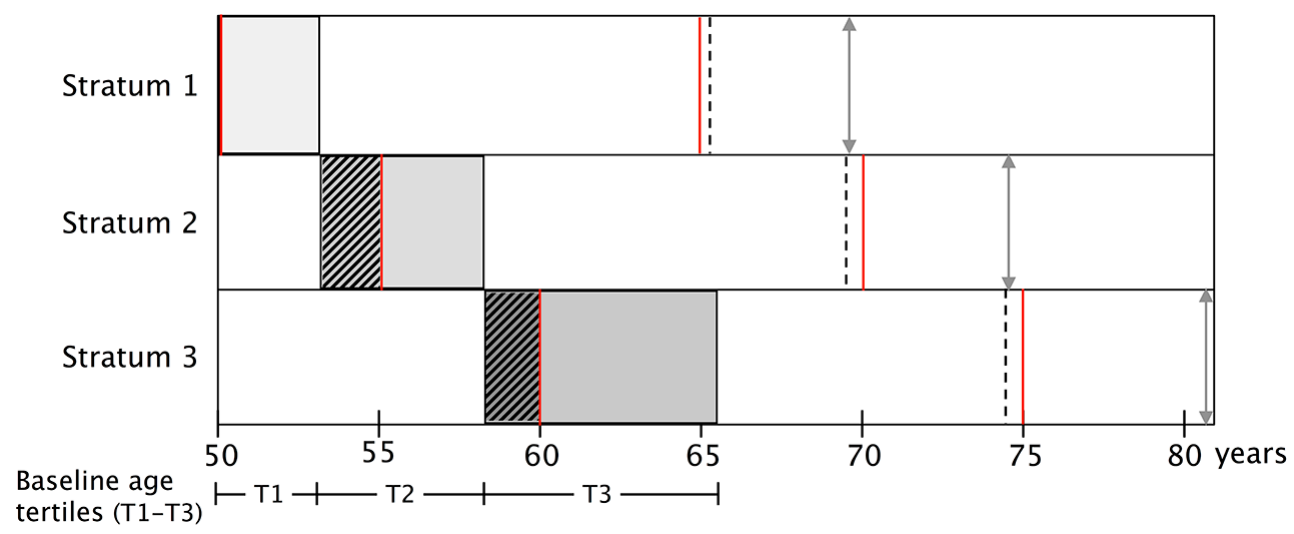

Figure 2: The three analysis strata based on baseline age tertiles (the baseline age span indicated by grey squares in three different tones. Red lines indicate analysis age spans. Dotted lines show median follow-up age. The number of observed persons diminishes close to maximum follow-up age which is indicated by grey lines with double-headed arrows. Participants leaving the study before the lower age limit in stratum 2 and 3 are not included in the analyses (the relevant age spans for this is shaded).

Age frames for cumulative risk: Subsequently to the decision regarding strata structure, we had to determine reasonable age frames for the cumulative risk for each stratum as this information were used in the further model control. This assessment relied on the distribution of follow-up time in each stratum. Thus, the specific choice of 15-year age frames was determined by the data. As illustrated in Figure 2, the follow-up time covers around 15 years of observation in each stratum (dotted lines indicate median follow-up age for each strata). For example, in stratum 1, the baseline age interval is 50-53.7 years and the median age at end of follow-up is 65.4 years (maximum age 69.6 years). Thus, the data in stratum 1 sufficiently cover an age frame up to 65 years.

Another applicable solution would have been to split the data set at specific ages (e. g. cutoffs at age 55 and 60 years). Due to a skewed distribution of baseline age in the Diet, Cancer and Health cohort, this would have resulted in strata of different sizes. We prioritized to have strata of equal size and therefore, we choose to stratify the data set according to baseline age tertiles.

The pseudo strata structure: When testing assumptions 1, 2, and 3 in the baseline age-stratified dataset, it turned out that many covariates were associated with baseline calendar time and baseline age indicating that assumption 3 was violated. This was addressed by a further stratification of each baseline age stratum into baseline calendar time tertiles, resulting in nine pseudo-observation strata (Table 3 ). Calendar time was chosen because it was a simple way of framing the covariate associations, and it worked due to a general calendar timecovariate association. We chose tertiles instead of for example quartiles or quintiles in order to have a sufficiently large number of subjects (more than 5500 individuals) in each pseudo-observation stratum, ensuring precision of the pseudo-observations. The same argument applies to our decision of tertile-based baseline age strata structure. Furthermore, it is also noticeable for that decision that strata with different start and end time points are not collapsible. So, for each stratum one analysis result is obtained (hence, Table 6 has three sets of results). This was also an argument for choosing the fewest possible number of baseline age strata.

Table 3: The nine pseudo-observation strata ${ }^{a}$.

\begin{tabular}{|c|c|c|c|}
\hline & $\begin{array}{l}\text { Baseline calendar time } \\
\text { 1st tertile } \\
(24 / 111993-20 / 10 \text { 1995) }\end{array}$ & $\begin{array}{l}\text { Baseline calendar time } \\
\text { 2nd tertile } \\
(21 / 101995-21 / 81996)\end{array}$ & $\begin{array}{l}\text { Baseline calendar time } \\
\text { 3rd tertile } \\
(22 / 81996-28 / 51997)\end{array}$ \\
\hline $\begin{array}{l}\text { STRATA } 1 \\
\text { Baseline age, } \\
\text { 1st tertile } \\
\quad \text { (age } 50.1-53.7)\end{array}$ & $\begin{array}{l}\text { Pseudo-observation } \\
\text { stratum } 1.1\end{array}$ & $\begin{array}{l}\text { Pseudo-observation } \\
\text { stratum } 1.2\end{array}$ & $\begin{array}{l}\text { Pseudo-observation } \\
\text { stratum } 1.3\end{array}$ \\
\hline $\begin{array}{l}\text { STRATA } 2 \\
\text { Baseline age, }\end{array}$ & $\begin{array}{l}\text { Pseudo-observation } \\
\text { stratum } 2.1\end{array}$ & $\begin{array}{l}\text { Pseudo-observation } \\
\text { stratum } 2.2\end{array}$ & $\begin{array}{l}\text { Pseudo-observation } \\
\text { stratum } 2.3\end{array}$ \\
\hline
\end{tabular}

2nd tertile

(age $53.7-58.8$ ) 
STRATA 3

Baseline age,

3rd tertile

(age $58.8-65.8)$

a Defined by baseline age and baseline calendar time tertiles. Minimum and maximum values for each tertile in parentheses. The pseudo-observation strata in each row were collapsed after generation of the pseudo-observations and before the GLM

Final tests of the independency assumptions: To check if assumption 1-4 were met in the suggested strata structure, the full model control was accomplished in each of the nine pseudo-observation strata by the procedure described in 3.3 .

Assumption 1: Except from pseudo-observation stratum 2.3 and 3.1, all strata fulfilled assumption 1. As the tests for stratum 2.3 and 3.1 were two of many statistical tests, the associations found may be due to chance. The amount of data could not support a more detailed stratification, as this would have led to insufficient coverage of the age frames for the analyses. Therefore, we decided to accept a deviation in the two pseudo-observation strata regarding assumption 1 .

Assumption 2: The main reason for incomplete follow-up was administrative censoring at a fixed calendar date (18,598 participants). Only 337 participants were censored due to loss to follow-up (emigration or change of personal identification number) and these could not be explored as no further information on these participants was available. The administrative part of the censoring was tested by expression (I). There was no association between AF and baseline calendar time. As assumption 1 was met to an acceptable degree, we concluded that age at administrative censoring was independent of AF risk during follow-up, hence the independent censoring assumption was met.

Assumption 3: Some statistically significant associations were found between covariates and baseline age or calendar time, but we assessed all of them to be too weak to be important in these analyses. Due to a large number of observations, the analyses were powered to detect also biologically irrelevant differences as being statistically significant. For example, in pseudo observation stratum 3.2, we found a statistically highly significant association between BMI and calendar time, but the association was weak (Table 4).

With a few minor exceptions, assumption 4 was met.

Conclusions from the model control and characteristic of each pseudo observation stratum is outlined in Table 5.

Data coverage. To avoid estimates with wide confidence intervals, it was checked if there was enough people at risk within each age interval. This was checked by Aalen-Johansen plots with confidence intervals made for each pseudo-observation strata. The confidence intervals were qualitatively assessed in the relevant analysis age interval (for pseudo-observation strata 2.1, 2.2, and 2.3 it was between age 55 and 70 years) (graphs not shown). Further, a high number of persons at risk $(>1000)$ at all events in all pseudo observation strata was found (Table 5).

Final strata structure: The pseudo-observation strata created by the baseline calendar time tertiles within each baseline age stratum were combined after generation of the pseudo-observations as they had the same start and end ages for cumulative risks. Hence, we ended up with three separate strata each covering a 15-year range with 10 years of overlap between strata (Figure 2). If diagnosed outside the relevant age range, cases were lost.

Table 4: Results of the test of assumption $3^{\mathrm{a}}$.

\begin{tabular}{|c|c|c|c|c|c|c|c|}
\hline \multicolumn{8}{|c|}{ A) Hypertension (dichotomous covariate) } \\
\hline Stratum $3(\mathrm{~N}=18,258)$ & $\begin{array}{l}\text { Hyper- } \\
\text { tension }\end{array}$ & $\begin{array}{c}\text { Baseline } \\
\text { age }^{\mathrm{b}}\end{array}$ & $95 \% \mathrm{CI}$ & $\begin{array}{r}P- \\
\text { value }^{\mathrm{c}}\end{array}$ & $\begin{array}{r}\text { Baseline } \\
\text { calendar } \\
\text { time }^{\mathrm{d}}\end{array}$ & $95 \%$ CI & $\begin{array}{r}P- \\
\text { value }^{\mathrm{c}}\end{array}$ \\
\hline $\begin{array}{l}\text { Pseudo-observation } \\
\text { stratum } 3.1 \\
\qquad(\mathrm{~N}=6,133)\end{array}$ & $\begin{array}{l}\text { No } \\
\text { Yes }\end{array}$ & $\begin{array}{l}61.93 \\
61.97\end{array}$ & $\begin{array}{l}61.87,61.98 \\
61.87,62.08\end{array}$ & 0.42 & $\begin{array}{l}31 / 31995 \\
28 / 31995\end{array}$ & $\begin{array}{l}27 / 3,5 / 4 \\
19 / 3,6 / 4\end{array}$ & 0.59 \\
\hline $\begin{array}{l}\text { Pseudo-observation } \\
\text { stratum } 3.2 \\
\quad(\mathrm{~N}=6,282)\end{array}$ & $\begin{array}{l}\text { No } \\
\text { Yes }\end{array}$ & $\begin{array}{l}61.84 \\
62.05\end{array}$ & $\begin{array}{l}61.79,61.89 \\
61.95,62.16\end{array}$ & $<0.001^{*}$ & $\begin{array}{l}15 / 31996 \\
17 / 31996\end{array}$ & $\begin{array}{l}13 / 3,18 / 3 \\
12 / 3,22 / 3\end{array}$ & 0.55 \\
\hline $\begin{array}{l}\text { Pseudo-observation } \\
\text { stratum } 3.3 \\
\quad(\mathrm{~N}=5,843)\end{array}$ & $\begin{array}{l}\text { No } \\
\text { Yes }\end{array}$ & $\begin{array}{l}61.81 \\
61.87\end{array}$ & $\begin{array}{l}61.76,61.87 \\
61.76,61.98\end{array}$ & 0.40 & $\begin{array}{l}26 / 121996 \\
23 / 121996\end{array}$ & $\begin{array}{l}24 / 12,28 / 12 \\
18 / 12,28 / 12\end{array}$ & 0.24 \\
\hline
\end{tabular}


B) BMI (continuous covariate)

\begin{tabular}{|c|c|c|c|c|c|c|c|}
\hline Stratum $3(\mathrm{~N}=18,258)$ & BMI & $\begin{array}{c}\text { Baseline } \\
\text { age }^{\mathrm{e}}\end{array}$ & $95 \% \mathrm{CI}$ & $\begin{array}{r}P_{-} \\
\text {value }^{\mathrm{c}}\end{array}$ & $\begin{array}{r}\text { Baseline } \\
\text { calendar } \\
\text { time }^{\mathrm{f}}\end{array}$ & $95 \% \mathrm{CI}$ & $\begin{array}{r}P- \\
\text { value }^{c}\end{array}$ \\
\hline $\begin{array}{l}\text { Pseudo-observation } \\
\text { stratum } 3.1 \\
\quad(\mathrm{~N}=6,133)\end{array}$ & & -0.003 & $-0.014,0.008$ & 0.61 & 0.073 & $-0.86,1.00$ & 0.88 \\
\hline $\begin{array}{l}\text { Pseudo-observation } \\
\text { stratum } 3.2 \\
\qquad(\mathrm{~N}=6,282)\end{array}$ & & 0.008 & $-0.004,0.020$ & 0.18 & 0.80 & $0.27,1.32$ & $<0.01^{* *}$ \\
\hline $\begin{array}{l}\text { Pseudo-observation } \\
\text { stratum } 3.3 \\
\qquad(\mathrm{~N}=5,843)\end{array}$ & & 0.008 & $-0.004,0.021$ & 0.19 & 0.45 & $-0.08,0.98$ & 0.10 \\
\hline \multicolumn{8}{|c|}{$\begin{array}{l}{ }^{*} \text { The statistical test is significant, but the association is small (approximately } 3 \text { months age difference between groups) } \\
\text { ** The statistical test is significant, but the association is small (for each step in BMI point, the mean inclusion date is 0.78 days later). } \\
\text { a Independency between A) hypertension and baseline age and baseline calendar time and B) BMI and baseline age and baseline calendar } \\
\text { time tested in crude linear regression analyses. Results from stratum } 3 \text { pseudo-observations strata }(3.1,3.2 \text { and 3.3) are shown } \\
\text { byean baseline age [y] } \\
\text { cWald test of equal estimates (two sided) } \\
{ }^{\mathrm{d}} \text { Mean baseline calendar date } \\
\text { e Mean change of baseline age [y] per BMI-point } \\
\text { f Mean change of baseline calendar time [days] per BMI-point }\end{array}$} \\
\hline
\end{tabular}

Table 5: Summary of the model control ${ }^{\mathrm{a}}$.

\begin{tabular}{|c|c|c|c|c|c|c|c|}
\hline \multirow[t]{2}{*}{ Analysis stratum ${ }^{b}$} & \multirow{2}{*}{$\begin{array}{l}\text { Pseudo ob- } \\
\text { servation } \\
\text { stratum }^{c}\end{array}$} & \multicolumn{2}{|c|}{ No at risk } & \multicolumn{4}{|c|}{ Model control assumptions } \\
\hline & & $\begin{array}{l}\text { at first } \\
\text { case }\end{array}$ & $\begin{array}{l}\text { by the end of } \\
\text { the age frame }\end{array}$ & 1 & 2 & 3 & 4 \\
\hline $\begin{array}{l}\text { Stratum } 1(50-65 \mathrm{y}) \\
\mathrm{N}=18,233 \\
\mathrm{~N}_{\text {cases }}=487\end{array}$ & $\begin{array}{l}1.1 \\
1.2 \\
1.3\end{array}$ & $\begin{array}{l}2687 \\
2296 \\
1331\end{array}$ & $\begin{array}{l}4896 \\
3668 \\
2217\end{array}$ & $\begin{array}{l}\text { Fulfilled } \\
\text { Fulfilled } \\
\text { Fulfilled }\end{array}$ & $\begin{array}{l}\text { Fulfilled } \\
\text { Fulfilled } \\
\text { Fulfilled }\end{array}$ & $\begin{array}{l}\text { Fulfilled } \\
\text { Fulfilled } \\
\text { Fulfilled }\end{array}$ & $\begin{array}{l}\text { Fulfilled } \\
\text { Fulfilled } \\
\text { Fulfilled }\end{array}$ \\
\hline $\begin{array}{l}\text { Stratum } 2(55-70 \text { y) } \\
N=18,202 \\
N_{\text {cases }}=705\end{array}$ & $\begin{array}{l}2.1 \\
2.2 \\
2.3\end{array}$ & $\begin{array}{l}2491 \\
2002 \\
1999\end{array}$ & $\begin{array}{l}3245 \\
2334 \\
1613\end{array}$ & $\begin{array}{l}\text { Fulfilled } \\
\text { Fulfilled } \\
\text { Baseline age } \\
\text { association }\end{array}$ & $\begin{array}{l}\text { Fulfilled } \\
\text { Fulfilled } \\
\text { Fulfilled }\end{array}$ & $\begin{array}{l}\text { Fulfilled } \\
\text { Fulfilled } \\
\text { Fulfilled }\end{array}$ & $\begin{array}{l}\text { Fulfilled } \\
\text { Fulfilled } \\
\text { Fulfilled }\end{array}$ \\
\hline $\begin{array}{l}\text { Stratum } 3(60-75 \text { y) } \\
N=18,258 \\
N_{\text {cases }}=1082\end{array}$ & $\begin{array}{l}3.1 \\
\\
3.2 \\
3.3\end{array}$ & $\begin{array}{l}1444 \\
1552 \\
1721\end{array}$ & $\begin{array}{l}3300 \\
2843 \\
2092\end{array}$ & $\begin{array}{l}\text { Baseline age } \\
\text { association } \\
\text { Fulfilled } \\
\text { Fulfilled }\end{array}$ & $\begin{array}{l}\text { Fulfilled } \\
\text { Fulfilled } \\
\text { Fulfilled }\end{array}$ & $\begin{array}{l}\text { Fulfilled } \\
\text { Fulfilled } \\
\text { Fulfilled }\end{array}$ & $\begin{array}{l}\text { Fulfilled } \\
\text { Fulfilled } \\
\text { Fulfilled }\end{array}$ \\
\hline
\end{tabular}

${ }^{a}$ The final model control was carried out within the nine pseudo-observation strata

${ }^{\mathrm{b}}$ The analysis strata 1,2 and 3 are based on baseline age tertiles

${ }^{\mathrm{c}}$ The intermediate pseudo-observation strata are based on baseline calendar time tertiles

\subsection{The independency issues in the diet, cancer and health cohort}

As mentioned in 3.3 it is important to distinguish between current age and baseline age. Age is a biological risk factor for AF opposed to baseline age which is a result of the cohort sampling - it simply states the age at which the analyst started to observe the participant, which solely relies on administrative decisions. The problem with baseline age in our cohort was that the persons that were invited to participate, when the cohort was established, seemed to be more willing to accept the invitation if they were healthier. So, a plausible explanation 
of the violation of assumption 1 is that the persons enrolled early in the baseline age interval (e. g. at age 50-55 years) accepted the invitation unaware of their future health status while the persons invited at older baseline ages (e. g. 60-65 years) had a tendency to refuse if they were in a poor health. One could imagine that a person invited at age 50 would accept the invitation (at that point in life, he/she would feel fit and healthy) while he would refuse had he been invited at an older age, where health symptoms had emerged. This selection problem at enrollment associated with baseline age could be termed a healthy participation problem. With regards to the observed associations between baseline calendar date and covariates in the model (risk factors for AF), this can be explained by a non-random selection during the inclusion period (1993-1997), where people living in areas with better socioeconomic characteristics were invited later in the recruiting period. We believe that both selection problems are common in observational cohort studies, but as long as they are accounted for in the development of the statistical model, valid analyses can still be obtained. The independency assumptions presented here are a prerequisite for generalisability of the results of the data analyses.

\subsection{Main result from the simplified setup}

Results from this simplified analysis are presented in Table 6 . We found no noticeable associations between $n-3$ and n- 6 and risk of AF, and no interactions between $n-3$ and n- 6 were found either.

Table 6: Intake of n-3 and n-6 LC-PUFAs and risk of atrial fibrillation analyzed by the pseudo-observation method with interaction assessed as deviation from additivity of risk differences. A: Stratum 1, B: Stratum 2, and C: Stratum $3^{\text {a }}$.

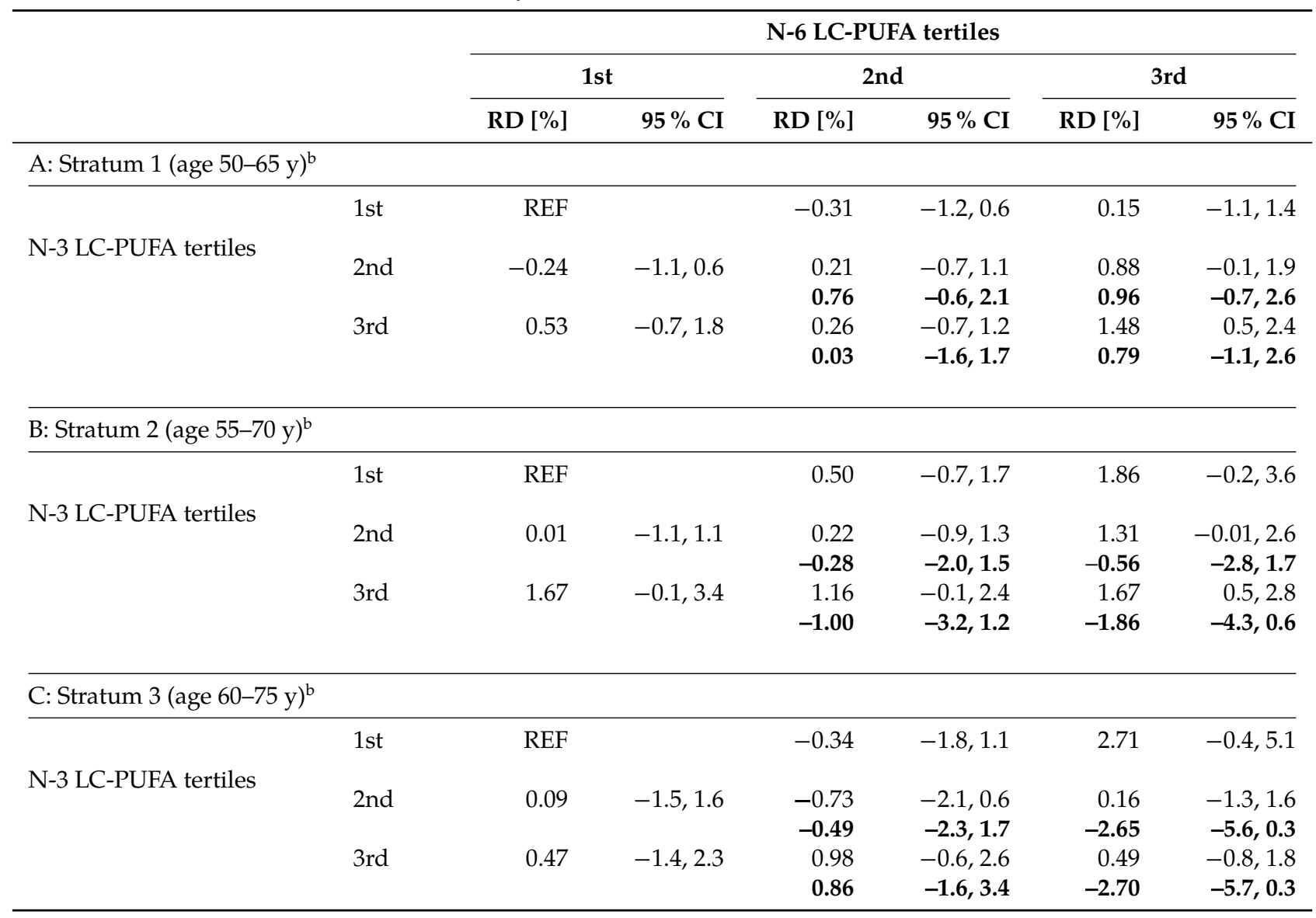

RD: Risk differences, LC-PUFA: Long-chain polyunsaturated fatty acid.

${ }^{\text {a }}$ The interaction analyses were performed for each stratum providing three parallel results in the table (A, B, and C). The ordinary font indicates the estimate of RD in each exposure group (nine per stratum) with the first n-3 and n-6 LC-PUFA tertile group as reference. The bold type indicates the estimate of interaction between n-3 and n- 6 assessed as the difference between the expected RD (based on additivity of the risk estimates for the individual exposures) and the observed RD in the combined exposure group. E. g. in the joint group of middle intake of n-3 and n-6 LC-PUFA in stratum 1 (Table 6A); the observed RD was $0.21 \%$ and the interaction was estimated to be $0.76 \%$ based on an expected RD of $-0.55 \%$ (calculated as the sum of -0.24 and -0.31 ). As the expected RDs were calculated according to RDs in individual groups (that is low intake of $n-3$ and middle/high intake of $n-6$ and vice versa), four estimates of interaction per strata were calculated. All RDs were adjusted for BMI and hypertension. Note that this table presents a simple model of the data and that the full analyses can be found elsewhere (Mortensen et al. 2017)

bStrata were defined by baseline age tertiles. In each stratum cumulative risk were measured in strata-specific 15 years age frames (given in parentheses) 


\section{Discussion}

The aim of this paper was to present the pseudo-observation method and explicitly demonstrate how to develop and validate the statistical model, which is an inevitable part of the analysis process. Although the model control in the pseudo-observation method is more comprehensive compared with Cox regression, the stratification approach in the pseudo-observation method makes it possible to handle violations. In Cox regression, on the other hand, the interpretation of the HR is not clear if the HR is not constant over time and the proportional hazards assumption is not met (Xu and $\mathrm{O}^{\prime}$ Quigley 2000).

The major advantage of the pseudo-observation approach is the possibility to carry out analyses using cumulative risks on both the additive and multiplicative risk scale, whereas Cox regression is limited to the multiplicative scale. The main aim of the cohort study, upon which the demonstration example presented here is based, was to explore potential biological interaction between two groups of LC-PUFAs. Based on Rothmans "sufficient cause diagrams" (Greenland, Lash, and Rothman 2008), it has been suggested in the literature that the relevant approach to assess interaction in public health contexts and in studies of biological factors in mechanistic interplay is to explore departure from additivity of absolute associations (Rothman, Greenland, and Walker 1980; Greenland, Lash, and Rothman 2008; VanderWeele and Knol 2014). Previously, other suggested approaches have been "Relative excess risk due to interaction" (RERI) (Li and Chambless 2007; VanderWeele 2011), additive hazards models (Rod et al. 2012) or additive interaction using survival percentiles (Bellavia, Bottai, and Orsini 2016).

In summary, the choice of method used for analyzing time-to-event data has often been the Cox proportional hazard regression, but the pseudo-observation method is a relevant alternative. We believe that the flexibility in the pseudo-observation method - in particular the possibility to operate on an absolute risk scale - counterbalances the extended model control, and that it is a relevant choice of method in analyses of cohort studies, especially if the interest is in investigating interaction from a public health point of view.

\section{Funding}

This article was supported by the Danish Council for Strategic Research (09-066965).

\section{References}

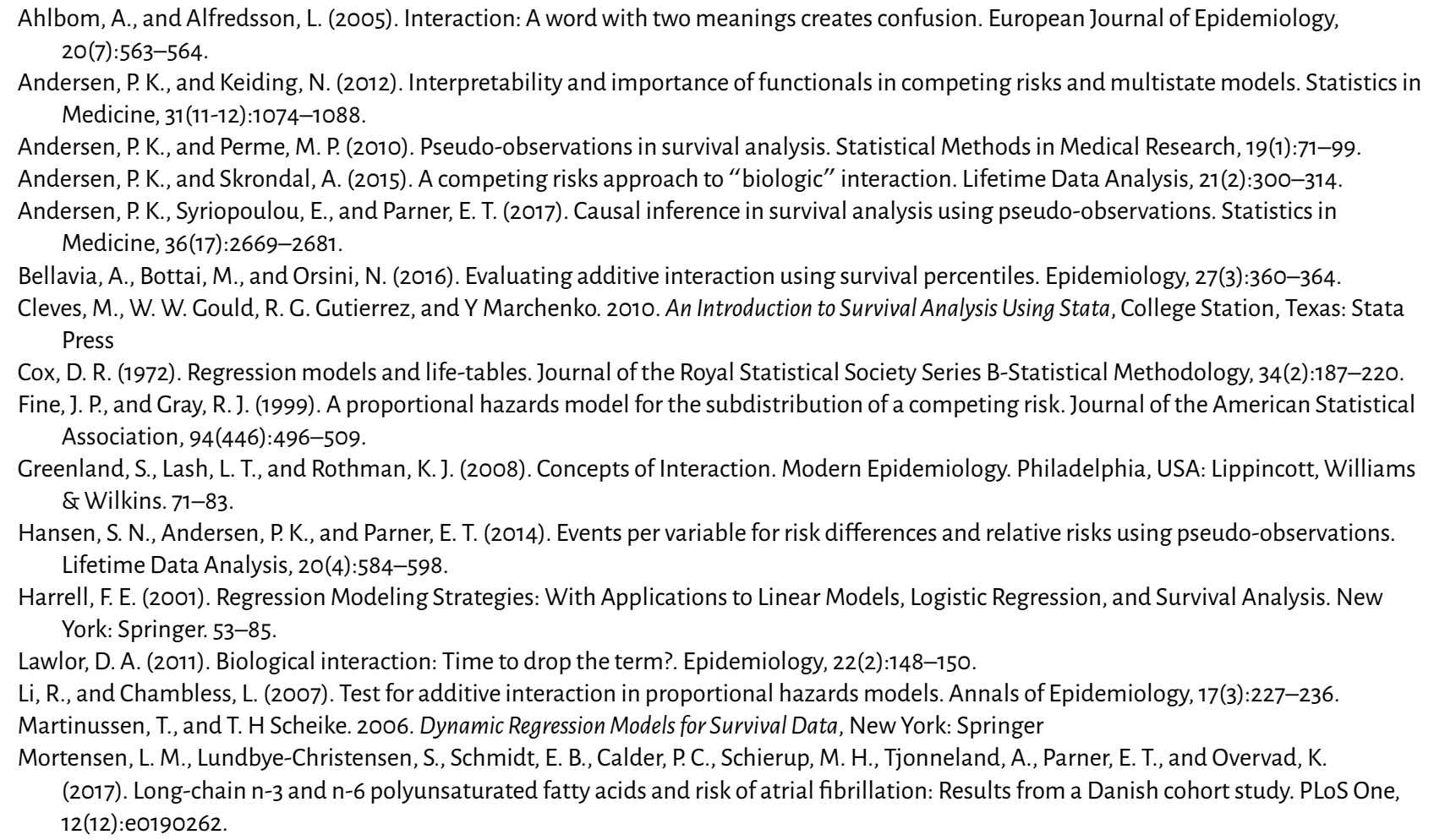


Overgaard, M., Andersen, P. K., and Parner, E. T. (2015). Regression analysis of censored data using pseudo-observations: An update. The Stata Journal, 15(3):809-821.

Overgaard, M., Parner, E. T., and Pedersen, J. (2017). Asymptotic theory of generalized estimating equations based on Jack-Knife pseudoobservations. Annals of Statistics, 45(5):1988-2015.

Parner, E. T., and Andersen, P. K. (2010). Regression analysis of censored data using pseudo-observations. The Stata Journal, 10(3):408-422.

Rod, N. H., Lange, T., Andersen, I., Marott, J. L., and Diderichsen, F. (2012). Additive interaction in survival analysis: Use of the additive hazards model. Epidemiology, 23(5):733-737.

Rothman, K. J., Greenland, S., and Walker, A. M. (1980). Concepts of interaction. American Journal of Epidemiology, 112(4):467-470.

StataCorp. (2013). Stata Statistical Software: Release 13. College Station, TX: StataCorp LP.

Tjonneland, A., Olsen, A., Boll, K., Stripp, C., Christensen, J., Engholm, G., and Overvad, K. (2007). Study design, exposure variables, and socioeconomic determinants of participation in diet, cancer and health: A population-based prospective cohort study of 57,053 men and women in Denmark. Scandinavian Journal of Public Health, 35(4):432-441.

VanderWeele, T. J. (2009). On the distinction between interaction and effect modification. Epidemiology, 20(6):863-871.

VanderWeele, T. J. (2011). Causal interactions in the proportional hazards model. Epidemiology, 22(5):713-717.

VanderWeele, T. J., and Knol, M. J. (2014). A tutorial on interaction. Epidemiologic Methods, 3(1):33-72.

Wu, N., Xu, B., Xiang, Y., Wu, L., Zhang, Y., Ma, X., Tong, S., Shu, M., Song, Z., Li, Y., and Zhong, L. (2013). Association of inflammatory factors with occurrence and recurrence of atrial fibrillation: A meta-analysis. International Journal of Cardiology, 169(1):62-72.

Xu, R., and O'Quigley, J. (2000). Estimating average regression effect under non-proportional hazards. Biostatistics, 1(4):423-439.

\section{Appendix, Stata syntax}

In the following, the variable 'event_date' denote the calendar date for events (diagnose of atrial fibrillation, competing risks, censoring) and 'event_status' denote the atrial fibrillation cases during follow-up.

\section{Assumption 1, test}

Here we examine the associations between baseline age (entry) and atrial fibrillation (event).

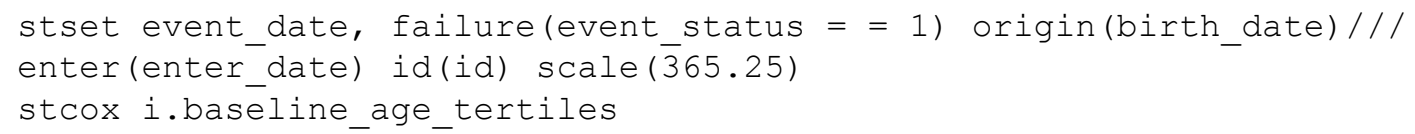

\section{Assumption 2, test}

Here we examine the associations between baseline date and atrial fibrillation (event).

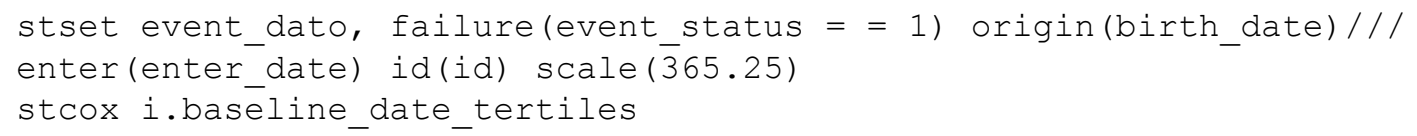

\section{Assumption 3, test}

Here we examine the associations between baseline age/baseline date (separately) and confounders in the GLM in univariate analyses ('i.' for a categorical confounder and 'c.' for a continuous confounder).






\section{Assumption 4, test}

Here we examine the associations between confounders in the GLM and censoring (administrative censoring during follow-up and lost to follow-up censoring) in univariate analyses.

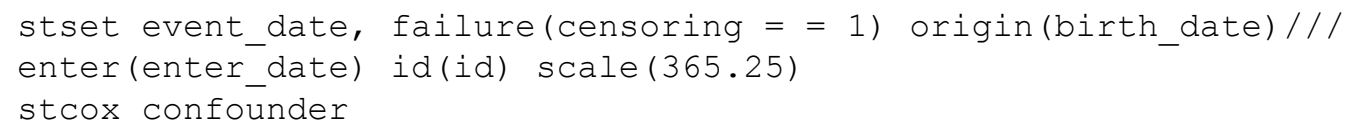

\section{Ceneration of pseudo-observations}

In the following example, the pseudo-observations are generated in stratum 1 consisting of three pseudoobservation strata (group 1-3), The time interval for cumulative risk is 15 years, start time: 50 year, end time: 65 year. The variable 'compet' denotes competing risk during follow-up.



The pseudo-observations (encoded in the variables: pseudo1, pseudo2 and pseudo3) are combined in the variable pseudo:

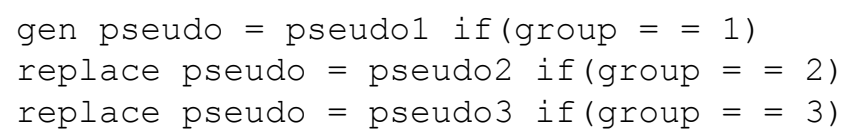

\section{The generalized linear modeling of the pseudo-observations}

Exposure: Interaction between intake of n-3 and n-6 LC-PUFAs ('n3' and 'n6'), modeled as tertiles.

Covariates: bmi (modeled as spline) and hypertension (dichotomous)

To obtain the risk differences in each tertile group ( $3^{*} 3$ groups) (the results presented in Table 6 ):

glm pseudo i.n3\#i.n6 bmi hypertension, link(id) vce(robust)

To obtain the estimates of interaction calculated as deviation from additivity of the risk differences (given with bold type in Table 6):

glm pseudo i.n3\#\#i.n6 bmi hypertension, link(id) vce(robust) 